\title{
Game-Based Virtual Reality Interventions to Improve Upper Limb Motor Function and Quality of Life After Stroke: Systematic Review and Meta-analysis
}

\author{
Pablo Domínguez-Téllez, MS, Jose A. Moral-Muñoz, PhD, , $^{2,3}$ Alejandro Salazar, PhD, ${ }^{3-5}$ \\ Esteban Casado-Fernández, MS, ${ }^{1}$ and David Lucena-Antón, $\mathrm{PhD}^{6}$
}

\begin{abstract}
Stroke is the main cause of disability in adulthood. Recent advances in virtual reality (VR) technologies have led to its increased use in the rehabilitation of stroke patients. A systematic review and meta-analysis of randomized controlled trials (RCTs) was conducted to determine the effectiveness of game-based reality on upper limb (UL) motor function and quality of life after stroke. In March 2018, a search of the following databases was performed: PubMed, PEDro, Web of Science, Scopus, The Cochrane Library, and Medline at EBSCO. The selection criteria were all RCTs published in English or Spanish during the past 10 years. The PEDro scale was used to evaluate the methodological quality of the studies. A total of 20 clinical trials were included in the systemic review, of which 15 contributed information to the meta-analysis. Favorable results were found for VR interventions on UL motor function (Fugl-Meyer Assessment for upper extremity, standardized mean difference $[\mathrm{SMD}]=1.53,95 \%$ CI $[0.51-2.54]$ ) and quality of life (functional independence measure, SMD $=0.77,95 \%$ CI [0.05-1.49]). The results demonstrate the potential benefits of VR interventions on the recovery of UL motor function and on quality of life after stroke.
\end{abstract}

Keywords: Virtual reality, Stroke, Physical therapy, Meta-analysis

\section{Introduction}

C erebrovascular diseases encompass a set of clinical neurological manifestations, which are caused by an intracranial or extracranial vascular alteration (arterial or venous). ${ }^{1}$ Among these conditions, cerebrovascular accidents, also known as strokes, are considered the leading cause of neurological disability in adults. ${ }^{2}$ Stroke represents the most common cause of physical disability in adults and the second most common cause of dementia. ${ }^{1}$ Further, most survivors experience motor disorders, associated with a reduction in quality of life $^{3}$ and the ability to perform activities of daily living (ADL). ${ }^{4}$

Neurorehabilitation involves a set of methods that aim at recovering neurological functions that were lost or diminished as a result of a brain or spinal damage. ${ }^{1}$ Although virtual reality (VR) started gaining popularity in the late 1980s and 1990s, VR technologies have only recently begun to be de- veloped and studied over the past decade as a possible tool for evaluation and treatment within this discipline. ${ }^{5}$ In addition to technological improvements in the systems, the drastic reduction in the cost of the devices, together with the widespread availability of high-speed internet connection, have led to an increase in their use. ${ }^{6}$ Accordingly, VR has reinvented itself in some technological areas, especially in videogames. These types of games have become popular across different commercial platforms, such as PlayStation, Xbox, Mac and PC. ${ }^{7}$

VR consists of the use of a user-computer interface that enables the real-time simulation of an environment, scenario, or activity, allowing the user to interact through multiple sensory canals. ${ }^{8,9}$ Currently, VR represents a multidisciplinary tool in clinical medicine, which is used in many applications including pain management, assessment of neurocognitive impairment, training of medical techniques, and physical rehabilitation. ${ }^{10}$ According to the research literature, VR is

\footnotetext{
${ }^{1}$ Centre Hospitalier Rives de Seine, Courbevoie, France.

${ }^{2}$ Department of Nursing and Physiotherapy, University of Cadiz, Cadiz, Spain.

${ }^{3}$ Institute of Research and Innovation in Biomedical Sciences of the Province of Cadiz (INiBICA), University of Cadiz, Cadiz, Spain.

${ }^{4}$ Department of Statistics and Operational Research, University of Cadiz, Cadiz, Spain.

${ }^{5}$ Observatory of Pain, Grünenthal Foundation-University of Cadiz, Cadiz, Spain.

${ }^{6}$ Department of Physiotherapy, University of Osuna, Seville, Spain.
} 
useful in visual, auditory, tactile, and motor learning applications; it has a positive effect on personal motivation, and it has also been applied to improve post-stroke motor skills. ${ }^{11}$ Research in this field has not only been limited to stroke rehabilitation; for example, one study used VR systems to evaluate motor levels after stroke. ${ }^{12}$

The term "virtual reality" includes a large number of technological devices and systems with different characteristics, which can be divided into two groups according to the level of patient immersion ${ }^{13}$ : (i) immersive systems, where users are fully integrated into the virtual environment with multisensory input through head-mounted displays, large screen projection, and VR caves; and (ii) semi-immersive or non-immersive systems, where a computer screen displays the environment, such as videogame consoles. These systems can also be combined with the use of treadmills, bionic gloves, and/or robot exoskeletons, which provide greater feedback to the user. VR devices allow for the creation of game-based interventions, in which it is possible to manipulate the content, duration, intensity, and feedback to create an adequate exer- cise prescription. Management of these training characteristics is closely related to recovery, reorganization, and neuroplasticity after stroke. ${ }^{2}$ Moreover, the main advantage of VR interventions over conventional treatment is that patients see it as a fun exercise game rather than treatment, leading to improvements in motivation and treatment adherence. ${ }^{9}$

Interventions with VR have also been demonstrated to improve functional mobility in children with cerebral palsy ${ }^{14}$ and in older adults. ${ }^{15}$ Likewise, its application has been evidenced in the re-training of walking and balance, as well as in the rehabilitation of upper limbs (UL) and lower limbs. ${ }^{11}$ However, there is not yet enough evidence for the effect of VR on traumatic brain injuries. ${ }^{16}$

Considering the current literature, more than a decade ago, in 2007, Henderson et al. ${ }^{13}$ concluded that the scientific evidence on VR in stroke rehabilitation was limited. Later, in 2014, Lohse et al. ${ }^{3}$ stated that VR therapy represents a significant advantage over conventional therapy in terms of body function and activity results, in addition to greater benefits of VR versus conventional therapy in relation to
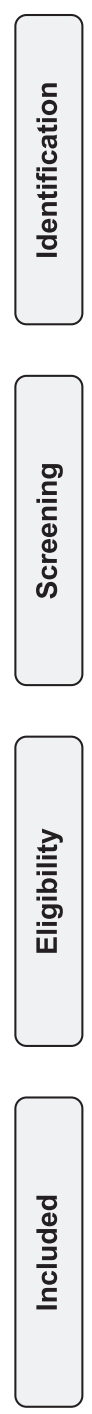
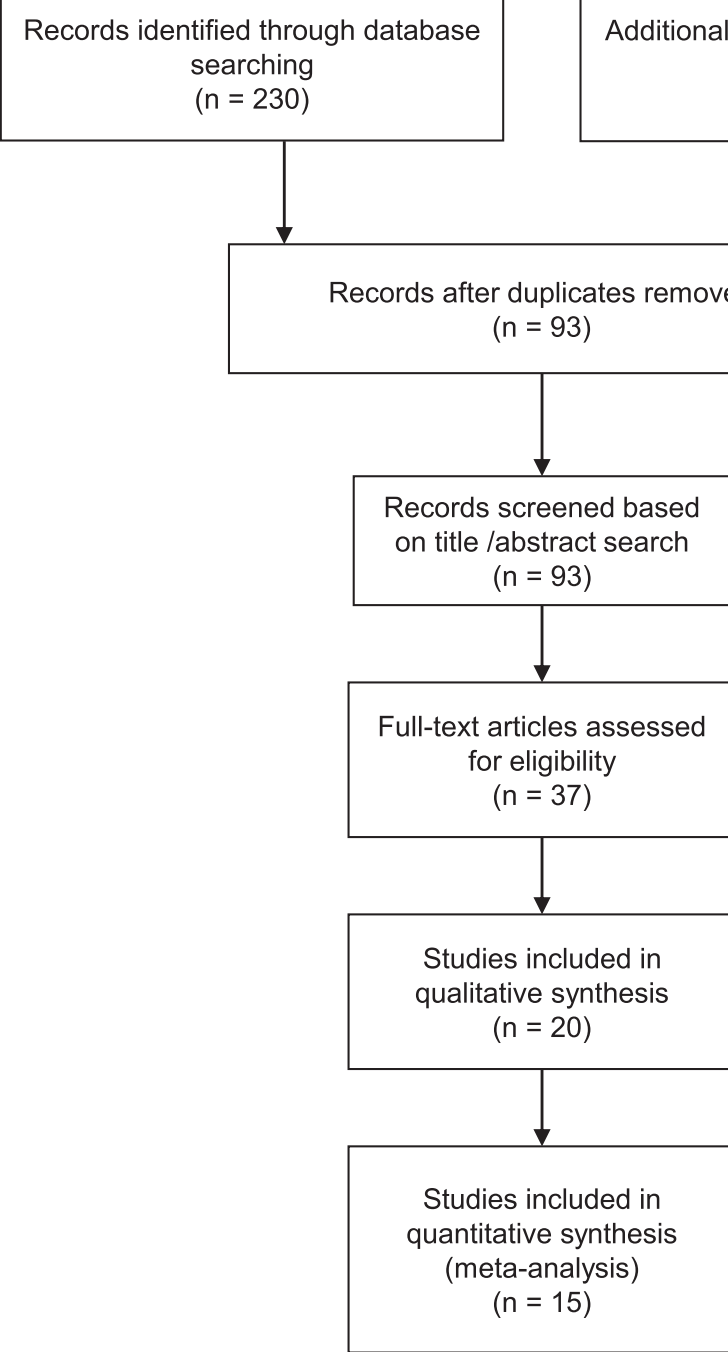

Additional records identified through other sources

$(n=0)$

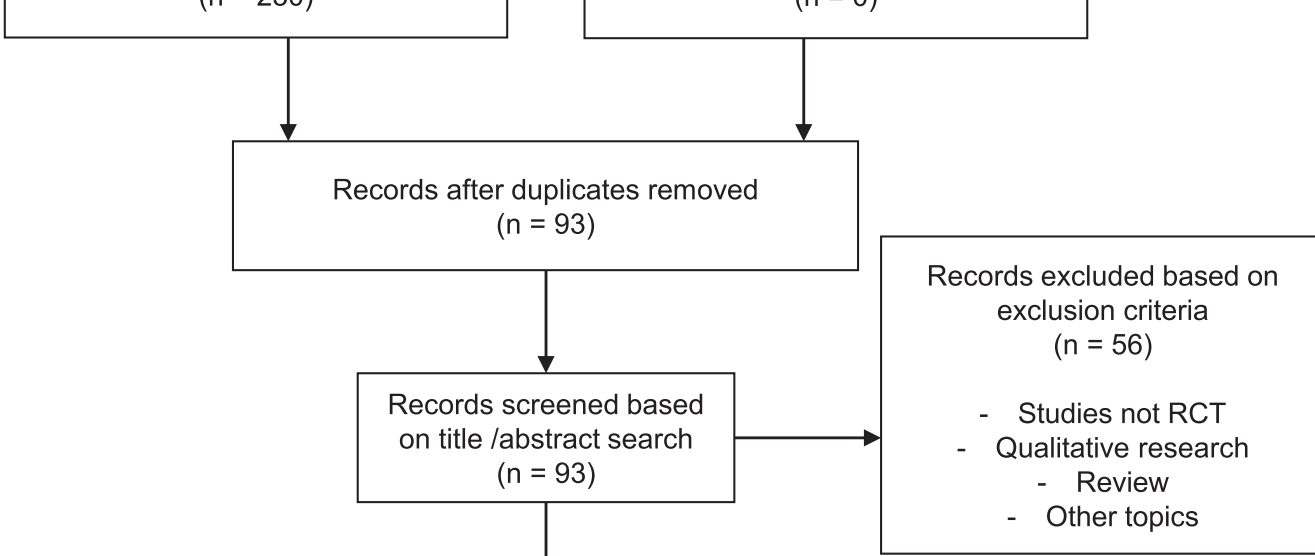

Full-text articles excluded $(n=17)$

- Not randomized

- Parameters not related to the targeted outcomes

FIG. 1. Information flow diagram of the different phases of the systematic review. 


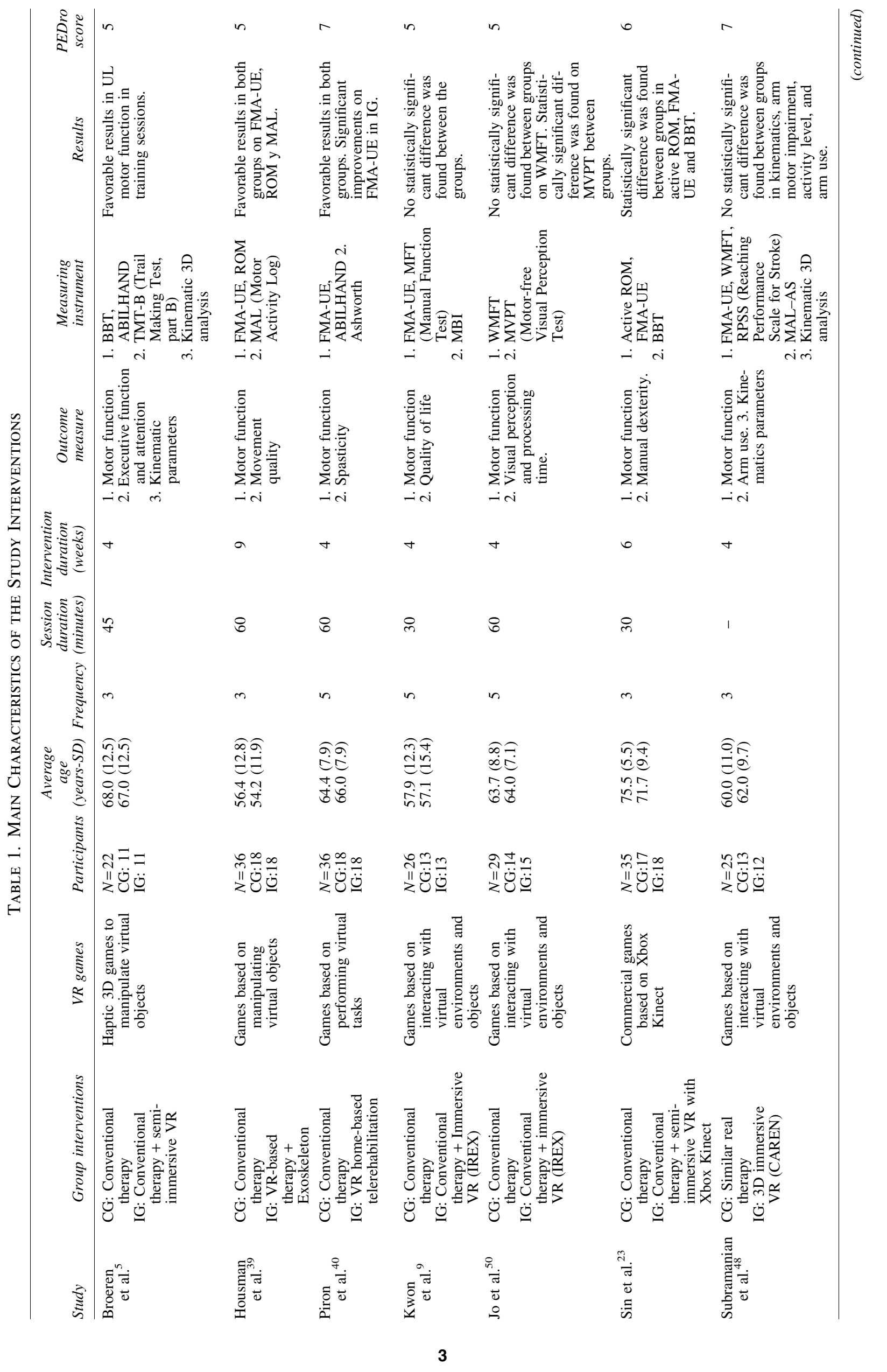




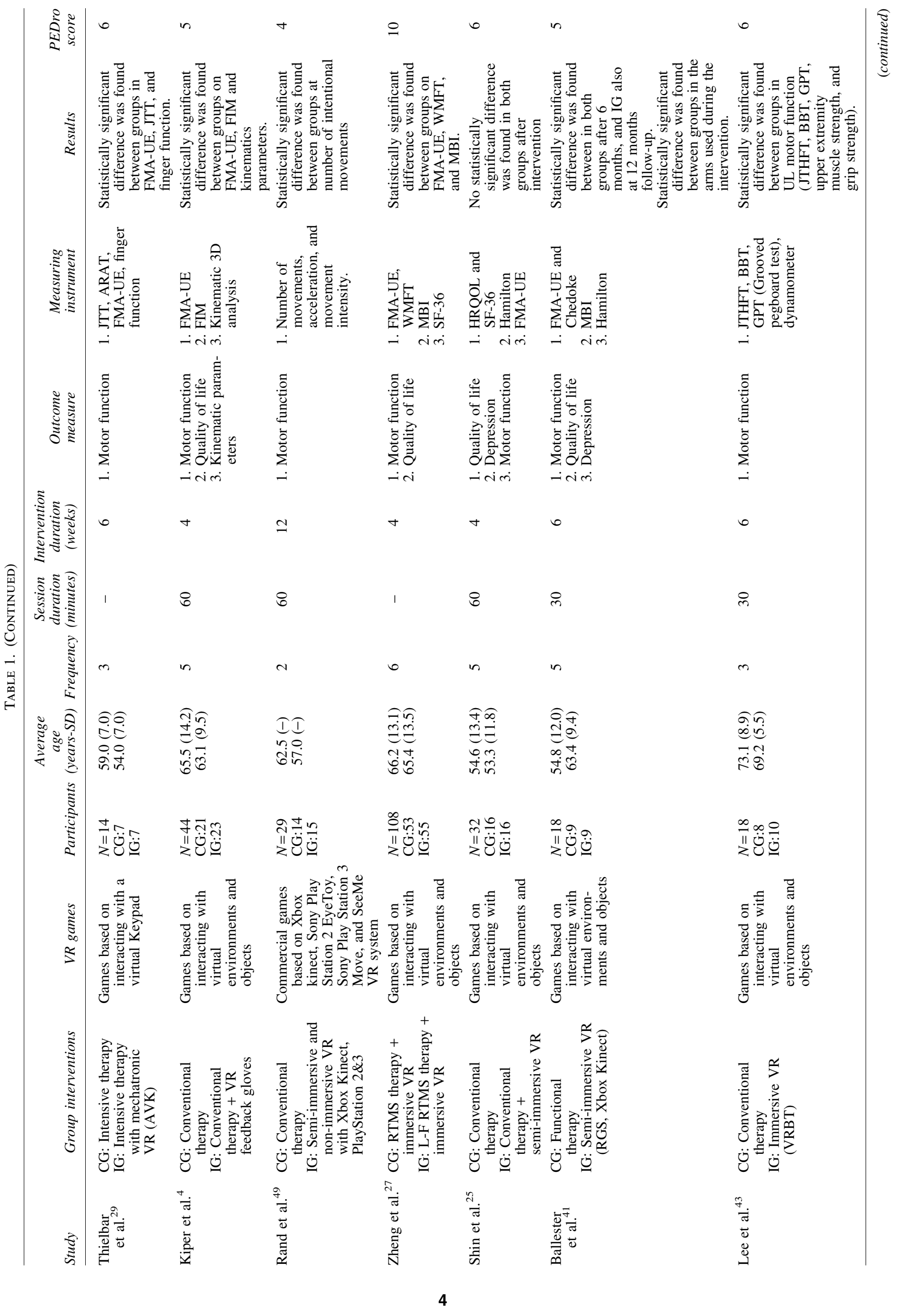




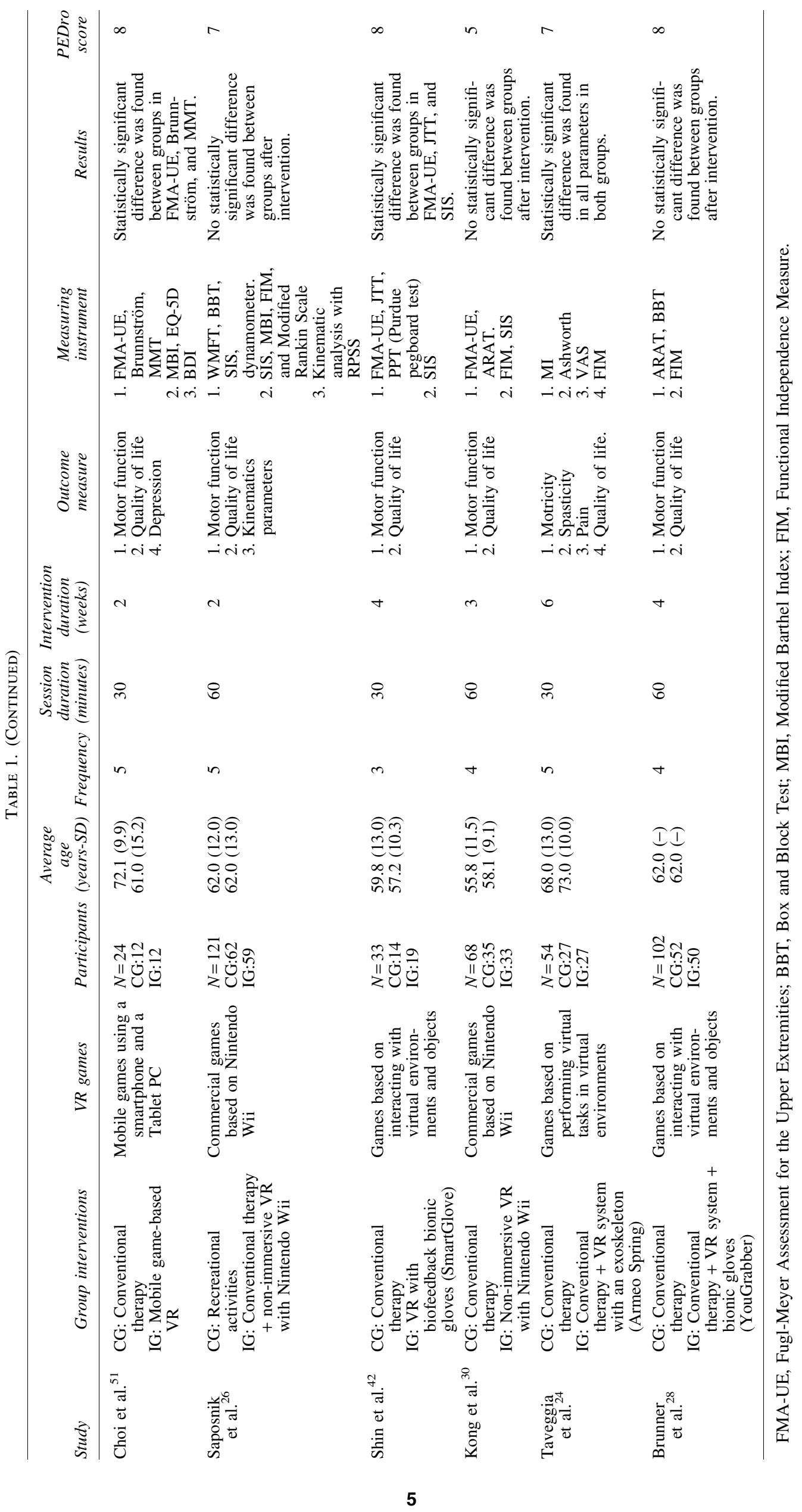


Table 2. Study Groups and Subgroups Included in the Meta-analysis

\begin{tabular}{|c|c|c|c|}
\hline$G$ & Studies & Outcome & Measuring instrument \\
\hline 1 & $\begin{array}{l}\text { Broeren et al.., }{ }^{5} \text { Housman et al. }{ }^{39} \text { Piron et al., }{ }^{40} \text { Kwon et al.., } \\
\text { Sin et al., }{ }^{23} \text { Thielbar et al. }{ }^{29} \text { Kiper et al.. }{ }^{4} \text { Rubio et al.,., } \\
\text { Lee et al., }{ }^{43} \text { Saposnik et al.., }{ }^{26} \text { Shin et al., }{ }^{42} \text { Kong et al., }{ }^{30} \text { Brunner et al. }{ }^{28}\end{array}$ & $\begin{array}{l}\text { UL Motor } \\
\text { Function }\end{array}$ & $\begin{array}{l}\text { FMA-UE } \\
\text { BBT }\end{array}$ \\
\hline 2 & $\begin{array}{l}\text { Kwon et al., }, \text { Zheng et al., }{ }^{27} \text { Rubio et al., }{ }^{41} \text { Saposnik et al.. }{ }^{26}{ }^{28} \\
\text { Kiper et al., }{ }^{4} \text { Kong et al., }{ }^{30} \text { Taveggia et al., }{ }^{24} \text { Brunner et al. }{ }^{28}\end{array}$ & Quality of life & $\begin{array}{l}\text { BARTHEL } \\
\text { FIM }\end{array}$ \\
\hline
\end{tabular}

treatment participation. These authors also stated that research in this field was limited. De Rooij et al. ${ }^{17}$ conducted a meta-analysis in 2016 that showed benefits on gait and balance from VR interventions in conjunction with conventional therapy. More recently, in 2017, a Cochrane review carried out by Laver et al. ${ }^{18}$ showed favorable results of VR in addition to conventional therapy on UL motor function, but that VR was comparable to conventional therapy. Considering this, our study aimed at analyzing the current scientific evidence of VR therapy for the recovery of the UL motor function and quality of life after stroke.

\section{Methods}

The present review was conducted and reported in line with the Preferred Reporting Items for Systematic Reviews and Meta-Analyses (PRISMA) guidelines for systematic reviews and meta-analyses. ${ }^{19}$

\section{Search strategy}

A search of the literature was performed in March 2018, using the following databases: PubMed, PEDro, Web of Science, Scopus, The Cochrane Library, and Medline EBSCO. Boolean search terms included the following: "stroke," "virtual reality," "virtual reality exposure therapy," and "physical therapy modalities." In PubMed, the searches were performed by using MeSH descriptors.

\section{Eligibility criteria}

The following inclusion criteria were established: (i) articles published in the last 10 years (2007-2018) to obtain the most relevant and updated research; (ii) English or Spanish language; (iii) randomized controlled trials (RCTs) as the study design; (iv) adult patients with stroke; (v) intervention based on games with immersive, semi-immersive, and non-immersive VR systems, simulating virtual environments using computers, video consoles, mobile apps and VR glasses; (vi) outcomes related to motor function of UL and quality of life. We excluded studies that combined participants with stroke with those with other kinds of conditions, which did not report the results separately.

\section{Study selection and data extraction}

First, we carried out a search combining keywords in different databases. Potentially relevant articles were identified after reading the title and abstract, and duplicated articles were eliminated. Subsequently, an exhaustive verification of compliance with the inclusion criteria was carried out to obtain the articles included in the present systematic review.

Two reviewers (D.L.A. and P.D.T.) actively participated in the process of selecting studies, reviewing and systematically extracting data from each study. A third reviewer (A.S.) participated in achieving consensus in case of different decisions. The following information was extracted from each article included in the review: authors, type of intervention, number of subjects, frequency of sessions per week, length of each session, total duration of the intervention, outcome measures, measurement instrument, and results obtained.

\section{Assessment of risk of bias}

The PEDro scale was used to evaluate the methodological quality of the RCTs included in the meta-analysis. ${ }^{20}$ It consists of 11 items related to the domains of selection, performance, detection, information, and attribution bases. ${ }^{21} \mathrm{~A}$ study with a score of 6 or more is considered as evidence level 1 (6-8 is good, 9-10 is excellent), and a study with a score of 5 or less is considered as evidence level 2 (4-5 is acceptable, $<4$ is poor). ${ }^{22}$

\section{Statistical analysis}

A meta-analysis was applied to compare changes in the effect size (pre- and post-intervention) between the intervention group (IG) and the control group (CG). Only metaanalyses with greater than or equal to four RCTs included

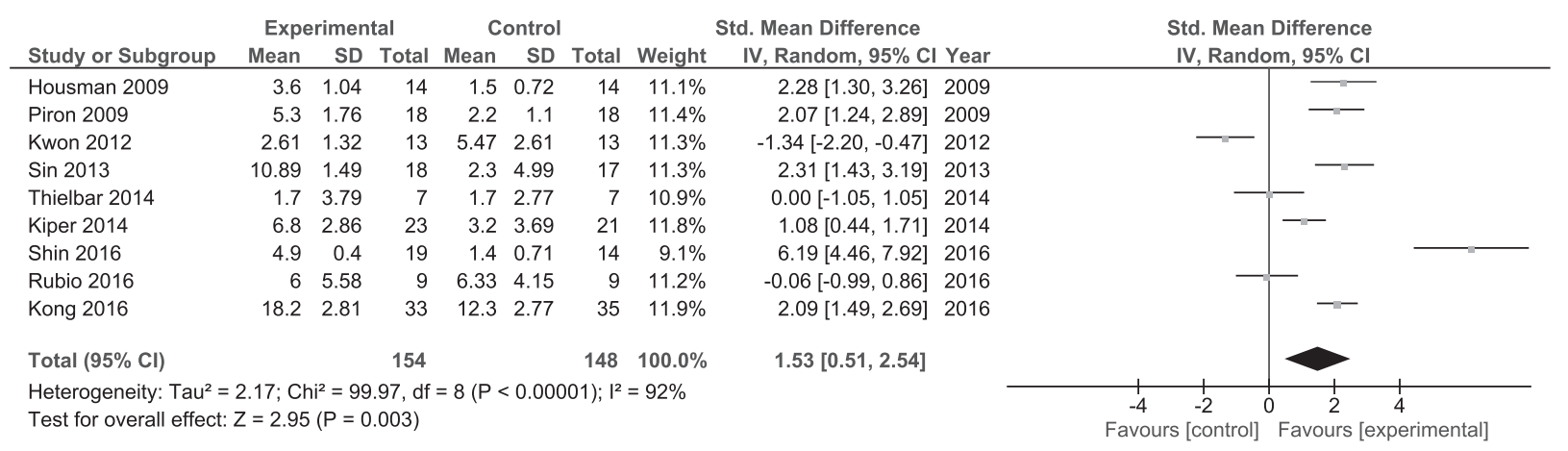

FIG. 2. Forest plot for FMA-UE. FMA-UE, Fugl-Meyer Assessment for the Upper Extremities. 


\begin{tabular}{|c|c|c|c|c|c|c|c|c|c|c|c|c|}
\hline \multirow[b]{2}{*}{ Study or Subgroup } & \multicolumn{3}{|c|}{ Experimental } & \multicolumn{3}{|c|}{ Control } & \multicolumn{3}{|c|}{ Std. Mean Difference } & \multirow{2}{*}{\multicolumn{3}{|c|}{$\begin{array}{l}\text { Std. Mean Difference } \\
\text { IV, Random, } 95 \% \mathrm{Cl}\end{array}$}} \\
\hline & Mean & SD & Total & Mean & SD & Total & Weight & IV, Random, 95\% Cl & Year & & & \\
\hline Broeren 2008 & 2.8 & 4.71 & 11 & 0.4 & 5.01 & 11 & $19.6 \%$ & $0.47[-0.38,1.32]$ & 2008 & & & \\
\hline $\operatorname{Sin} 2013$ & 9.56 & 3.07 & 18 & 2.7 & 2.65 & 17 & $19.4 \%$ & $2.33[1.45,3.21]$ & 2013 & & & \\
\hline Saposnik 2016 & 4.3 & 1.78 & 71 & 6.7 & 1.64 & 70 & $21.2 \%$ & $-1.39[-1.76,-1.03]$ & 2016 & - - & & \\
\hline Lee, S 2016 & 14.19 & 5.54 & 10 & 6.13 & 4.89 & 8 & $18.5 \%$ & $1.46[0.38,2.53]$ & 2016 & & & \\
\hline Brunner 2017 & 11.8 & 2.15 & 62 & 11.5 & 2.28 & 58 & $21.3 \%$ & $0.13[-0.22,0.49]$ & 2017 & & - & \\
\hline Total $(95 \% \mathrm{CI})$ & & & 172 & & & 164 & $100.0 \%$ & $0.55[-0.66,1.76]$ & & & & \\
\hline $\begin{array}{l}\text { Heterogeneity: } \mathrm{Tau}^{2}= \\
\text { Test for overall effect }\end{array}$ & $\begin{array}{l}1.75 \\
z=0\end{array}$ & $\begin{array}{l}\mathrm{hi}^{2}= \\
9(\mathrm{P}=\end{array}$ & $\begin{array}{l}86.83, \mathrm{~d} \\
0.37)\end{array}$ & $\mathrm{df}=$ & . & $0001)$ & $; I^{2}=95 \%$ & & & $\begin{array}{l}-2 \\
\text { Favours }\end{array}$ & $\stackrel{0}{\text { rol] Favou }}$ & ${ }_{\text {peri }}^{2}$ \\
\hline
\end{tabular}

FIG. 3. Forest plot for BBT. BBT, Box, and Block Test.

were considered to increase the confidence in our findings. For the meta-analysis, the standardized mean difference was calculated along with the $95 \%$ confidence interval (CI), and the significance level was set at $P<0.05$. Heterogeneity was determined by the chi-square test and the $I^{2}$ statistic. When homogeneity was observed, a fixed-effect model was used. In the case of heterogeneity, a random-effects model was used. The results were presented in Forest plots. All statistical analyses were carried out by using the statistical software Review Manager (RevMan) 5.3 (The Cochrane Collaboration, The Nordic Cochrane Centre).

\section{Results}

As illustrated in Figure 1, the database searches were carried out by using the aforementioned combination of keywords, which retrieved a total of 230 documents. After the removal of duplicates, 93 articles were screened for eligibility. After the selection process, a total of 20 studies were included in the review, of which 15 were included in the meta-analysis for the statistical comparison. The entire selection process in the corresponding phases is detailed in Figure 1.

\section{Risk of bias}

The methodological quality of the included studies was generally high (average total PEDro score $=6.25$, range 410). The score obtained as well as the detailed characteristics of each study are shown in Table 1.

\section{Data extraction}

A total of 874 subjects (CG, $n=434$; IG with VR, $n=440$ ) participated in the included studies. Regarding participant age, the highest mean age among the CG belonged to the study by Sin and Lee, ${ }^{23}$ with 75.5 years, whereas the highest among the IG belonged to the study by Taveggia et al., ${ }^{24}$ with 73.0 years. The lowest ages for CG and IG appeared in the study by Shin et al., ${ }^{25}$ with 54.6 and 53.3 years, respec- tively. Considering the number of participants, three studies stand out with samples composed of more than 100 subjects: Saposnik et al., ${ }^{26}$ Zheng et al., ${ }^{27}$ and Brunner et al. ${ }^{28}$ The remaining studies recruited between $14^{29}$ and $68^{30}$ subjects, with a similar distribution of subjects between CG and IG. Table 1 shows the main characteristics of the interventions carried out in the different studies.

\section{Study groups and subgroups included in the meta-analysis}

Table 2 shows the characteristics of the different groups created for the meta-analysis. The physical outcomes analyzed in the studies were considered for the creation of these groups. Accordingly, two groups were established: (i) UL motor function and (ii) quality of life. Moreover, different subgroups were created depending on the instrument used to measure the outcomes. In this way, the UL motor function group and the quality of life group were each further divided into two subgroups.

The results of the meta-analysis are presented in Figures $2-5$. In the UL motor function group, two different instruments were used: Fugl-Meyer Assessment for the Upper Extremities (FMA-UE), and Box and Block Test (BBT). The FMA-UE scale is a widely used evaluation system for hemiplegic patients that measures stroke-related motor impairment. ${ }^{31}$ It includes different items that assess movement, coordination, and reflex action from the shoulder to hands, ${ }^{32}$ and it has been confirmed to have high validity and reliability. ${ }^{33}$ For FMA-UE, the overall result of the metaanalysis was favorable. On the other hand, the BBT is used to measure gross manual dexterity. It consists of asking the patient to move as many small wooden blocks as possible from one box to another in 1 minute. ${ }^{34}$ The overall result of the meta-analysis was not conclusive.

Finally, the quality of life group was assessed by using two instruments: Modified Barthel Index (MBI), and Functional Independence Measure (FIM). The MBI is a scale to measure the degree of functional independence, with scores ranging

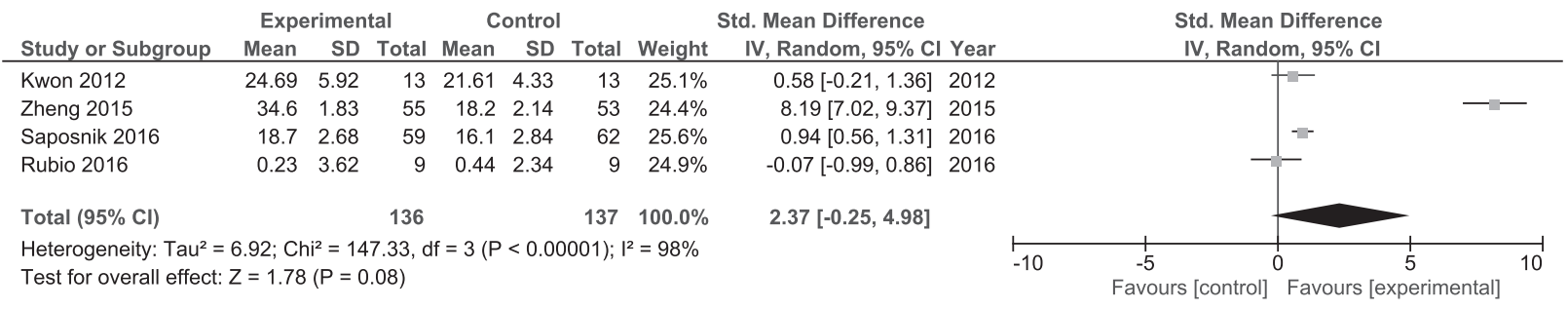

FIG. 4. Forest plot for the quality of life. MBI, Modified Barthel Index. 


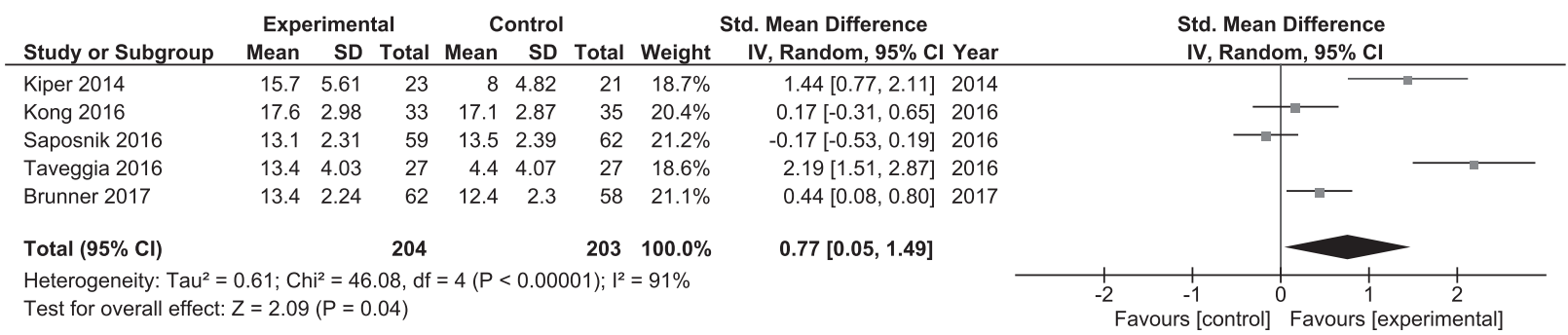

FIG. 5. Forest plot for the quality of life. FIM, Functional Independence Measure.

from 0 to $100 .{ }^{35}$ The overall result in the meta-analysis was not conclusive. The FMI is a scale to measure the severity of the patient's disability. ${ }^{36}$ The overall result was favorable.

\section{Discussion}

The aim of this systematic review and meta-analysis of RCTs was to analyze the efficacy of different types of VR on improving UL motor function and quality of life after stroke.

VR-based systems have many advantages over other interventions, making them a potential physical intervention tool. These advantages include their low cost, easy portability, and compatibility with other systems, in addition to the increased motivation of participants through the use of games, and the ability to provide direct feedback to the patient. ${ }^{37}$ Moreover, the application of VR through home-based interventions could be effective in stroke rehabilitation. ${ }^{38}$

Regarding the specific technologies used by different studies that obtained favorable results, the following were employed: an innovative exoskeleton, ${ }^{24,39}$ VR telerehabilitation system, ${ }^{40}$ IREX immersion VR systems, ${ }^{9,27}$ Xbox Kinect, ${ }^{23,41}$ keyboard with VR, ${ }^{29}$ VR combined with gloves, ${ }^{4,28,42}$ Nintendo Wii, ${ }^{26,30}$ and virtual surfaces. ${ }^{43}$ All of these technologies share common characteristics, such as the interaction of the user with a virtual environment by playing games, in which the subject acts in a similar way to reality, depending on the level of immersion used. In this way, the higher the immersion is, the less contact they have with the external physical world, thereby enhancing the user's attention. It is important to note the negative effect of attention problems, which are the most prominent neuropsychological issue after stroke,$^{44}$ on learning. Therefore, the use of immersive VR systems, which helps the user focus their attention on the task entirely, could confer great advantages in the recovery of deficits.

The statistical analysis carried out in the present review revealed favorable results of VR intervention on the motor function of UL, especially in the full FMA-UE. Our results are consistent with those of Laver et al., ${ }^{18}$ who reported that VR interventions could be effective when used in conjunction with conventional therapy for improving UL motor function and ADL after stroke. However, it would be interesting to analyze which specific components of therapy make it possible to achieve these positive effects. Along these lines, therapy through VR includes four components that could work together to ensure success: intensive therapy, motivating therapy through exercise games, stimulation of motor learning, and positive feedback between the stimulus and the response. ${ }^{45}$

The findings on quality of life highlight the potential benefits of VR interventions after stroke. According to the International Classification of Functioning, Disability and
Health (ICF) ${ }^{46}$ participation restrictions could be influenced by activity limitations, which can be influenced by impairments at the functional level and by body structure. However, there is not always a direct relationship between these three domains. ${ }^{47}$ We can suggest that the improvements in UL function revealed in the present review could improve the participation in ADL and, consequently, lead to improvements in quality of life. Further, the improvements obtained in the FIM scale could be related to those obtained in the FMA-UE scale, as there is known to be a correlation between both instruments. ${ }^{32}$ Following the line of research proposed by Lohse et al., ${ }^{3}$ we encourage researchers to state which domains of the ICF scale they intend to improve through the intervention.

Finally, according to Pietrzak et al., ${ }^{16}$ emphasis should be placed on the need for incorporating videogames based on VR systems into stroke rehabilitation, in addition to making the service easier to use for therapy centers and home-based treatments. Moreover, therapists could design individualized VR games based on the patient's clinical features, by changing the duration, intensity, and difficulty level, and also by providing different types of feedback and positive reinforcements. ${ }^{38}$

Although this article presents relevant information, some limitations need to be addressed. One limitation was related to the great variety of VR interventions, all of which are grouped under the same term or keyword. Further, acute and chronic strokes were not analyzed separately. Some studies $^{25,48-51}$ were not included in the meta-analysis as they did not provide the data collected before or after the intervention. Another limitation was the different measuring instruments. It was not possible to statistically compare different studies because different versions of the same scale or different measuring units were used.

\section{Conclusions}

According to the results obtained, we can conclude that VR seems to be effective for the improvement of motor function of UL and quality of life after stroke.

This study can be helpful in clinical practice through the use of videogames based on VR systems and as a preliminary step for the realization of new studies on the subject, as well as by providing constructive criticism to improve the methodology of future interventions. It will be necessary to conduct clinical trials that use larger sample sizes and greater homogeneity in terms of the device used, the frequency and the intensity of the interventions, as well as trying to identify which specific factors of therapy have a greater weight in achieving a positive outcome. 


\section{Author Disclosure Statements}

No competing financial interests exist.

\section{References}

1. Murie-Fernández M, Irimia P, Martínez-Vila E, Meyer MJ, Teasell R. Neuro-rehabilitation after stroke. Neurologia 2010; 25:189-196.

2. Federación Española de Ictus. Sant Voi de Llobregat. 2018. https://www.ictusfederacion.es (accessed March 17, 2018).

3. Lohse KR, Hilderman CGE, Cheung KL, et al. Virtual reality therapy for adults post-stroke: A systematic review and meta-analysis exploring virtual environments and commercial games in therapy. PLoS One 2014; 9:e93318.

4. Kiper PB, Agostini M, Luque-Moreno C, et al. Reinforced feedback in virtual environment for rehabilitation of upper extremity dysfunction after stroke: Preliminary data from a randomized controlled trial. Biomed Res Int 2014; 2014: $1-8$.

5. Broeren J, Claesson L, Goude D, et al. Virtual rehabilitation in an activity centre for community-dwelling persons with stroke. Cerebrovasc Dis 2008; 26:289-296.

6. Merchant Z, Goetz ET, Cifuentes L, et al. Effectiveness of virtual reality-based instruction on students' learning outcomes in K-12 and higher education: A meta-analysis. Comput Educ 2014; 70:29-40.

7. Virtual Reality Society. When Was Virtual Reality Invented? United Kingdom. 2017. https://www.vrs.org.uk/ virtual-reality/invention.html (accessed March 17, 2018).

8. Adamovich S V, Fluet GG, Tunik E, Merians AS. Sensoriomotor training in virtual reality: A review. NeuroRehabilitation 2009; 25:29-44.

9. Kwon J-S, Park M-J, Yoon I-J, Park S-H. Effects of virtual reality on upper extremity function and activities of daily living performance in acute stroke: A double-blind randomized clinical trial. NeuroRehabilitation 2012; 31: 379-385.

10. Pourmand A, Davis S, Lee D, et al. Emerging utility of virtual reality as a multidisciplinary tool in clinical medicine. Games Health J 2017; 6:263-270.

11. Park Y-H, Lee C, Lee B-H. Clinical usefulness of the virtual reality-based postural control training on the gait ability in patients with stroke. J Exerc Rehabil 2013; 9: 489-494.

12. Kim W, Cho S, Baek D, et al. Upper Extremity functional evaluation by Fugl-Meyer assessment scoring using depthsensing camera in hemiplegic stroke patients. PLoS One 2016; 11:e0158640.

13. Henderson A, Korner-bitensky N, Levin M. Virtual reality in stroke rehabilitation: A systematic review of its effectiveness for upper limb motor recovery. Top Stroke Rehabil 2007; 14:52-61.

14. Brien $M$, Sveistrup H. An intensive virtual reality program improves functional balance and mobility of adolescents with cerebral palsy. Pediatr Phys Ther 2011; 23:258-266.

15. Bisson E, Contant B, Sveistrup H, Lajoie Y. Functional balance and dual-task reaction times in older adults are improved by virtual reality and biofeedback training. Cyberpsychol Behav 2007; 10:16-23.

16. Pietrzak E, Pullman S, McGuire A. Using virtual reality and videogames for traumatic brain injury rehabilitation: A structured literature review. Games Health J 2014; 3: 202-214.
17. de Rooij IJM, van de Port IGL, Meijer J-WG. Effect of virtual reality training on balance and gait ability in patients with stroke: Systematic review and meta-analysis. Phys Ther 2016; 96:1905-1918.

18. Laver KE, Lange B, George S, et al. Virtual reality for stroke rehabilitation. Cochrane Database Syst Rev 2017; 11:CD008349.

19. Hutton B, Catalá-lópez F, Moher D. The PRISMA statement extension for systematic reviews incorporating network meta-analysis: PRISMA-NMA. Med Clin (Barc) 2016; 147:262-266.

20. Bhogal SK, Teasell RW, Foley NC, Speechley MR. The PEDro scale provides a more comprehensive measure of methodological quality than the Jadad Scale in stroke rehabilitation literature. $\mathrm{J}$ Clin Epidemiol 2005; 58: 668-673.

21. Verhagen AP, Vet HCW De, Bie RA De, et al. The Delphi List: A criteria list for quality assessment of randomized clinical trials for conducting systematic reviews developed by Delphi Consensus. J Clin Epidemiol 1998; 51:12351241.

22. Foley NC, Teasell RW, Bhogal SK, Speechley MR. Stroke rehabilitation evidence-based review: Methodology. Top Stroke Rehabil 2003; 10:1-7.

23. Sin $\mathrm{H}$, Lee $\mathrm{G}$. Additional virtual reality training using Xbox Kinect in stroke survivors with hemiplegia. Am J Phys Med Rehabil 2013; 92:871-880.

24. Taveggia G, Borboni A, Salvi L, et al. Efficacy of robotassisted rehabilitation for the functional recovery of the upper limb in post-stroke patients: A randomized controlled study. Eur J Phys Rehabil Med 2016; 52:767-773.

25. Shin J-H, Park SB, Jang SH. Effects of game-based virtual reality on health-related quality of life in chronic stroke patients: A randomized, controlled study. Comput Biol Med 2015; 63:92-98.

26. Saposnik G, Cohen LG, Mamdani M, et al. Efficacy and safety of non-immersive virtual reality exercising in stroke rehabilitation (EVREST): A randomised, milticentre, single-blind, controlled trial. Lancet Neurol 2016; 15:1019-1027.

27. Zheng C, Liao W, Xia W. Effect of combined lowfrequency repetitive transcranial magnetic stimulation and virtual reality training on upper limb function in subacute stroke: A double-blind randomized controlled trail. J Huazhong Univ Sci Technol Med Sci 2015; 35:248-254.

28. Brunner I, Skouen JS, Hofstad H, et al. Virtual Reality Training for Upper Extremity in Subacute Stroke (VIRTUES): A multicenter RCT. Neurology 2017; 89:24132421.

29. Thielbar KO, Lord TJ, Fischer HC, et al. Training finger individuation with a mechatronic-virtual reality system leads to improved fine motor control post-stroke. J Neuroeng Rehabil 2014; 11:171.

30. Kong K, Loh Y, Thia E, et al. Efficacy of a virtual reality commercial gaming device in upper limb recovery after stroke: A randomized, controlled study. Top Stroke Rehabil 2016;23:333-340.

31. Fugl-Meyer AR, Jaasko L, Leyman I, et al. The post-stroke hemiplegic patient. 1. A method for evaluation of physical performance. Scand J Rehabil Med 1975; 7:13-31.

32. Bürge E, Kupper D, Finckh A, et al. Neutral functional realignment orthosis prevents hand pain in patients with subacute stroke: A randomized trial. Arch Phys Med Rehabil 2008; 89:1857-1862. 
33. Rabadi MH, Rabadi FM. Comparison of the action research arm test and the Fugl-Meyer assessment as measures of upper-extremity motor weakness after stroke. Arch Phys Med Rehabil 2006; 87:962-966.

34. Mathiowetz V, Volland G, Kashman N, Weber K. Adult norms for the box and block test of manual dexterity. Am J Occup Ther 1985; 39:386-391.

35. Collin C, Wade D, Davies S, Horne V. The Barthel ADL Index: A reliability study. Int Disabil Stud 1987; 10:61-63.

36. Linacre JM, Heinemann AW, Wright BD, et al. The structure and stability of the functional independence measure. Arch Phys Med Rehabil 1994; 75:127-132.

37. Ruiz-González L, Lucena-Antón D, Salazar A, et al. Physical therapy in Down syndrome: Systematic review and meta-analysis. J Intellect Disabil Res 2019; 68(3):10411067.

38. Lin J, Kelleher CL, Engsberg JR. Developing home-based virtual reality therapy interventions. Games Health J 2013; 2:34-38.

39. Housman SJ, Scott KM, Reinkensmeyer DJ. A randomized controlled trial of gravity-supported, computer-enhanced arm exercise for individuals with severe hemiparesis. Neurorehabil Neural Repair 2009; 23:1505-1514.

40. Piron L, Turolla A, Agostini M, et al. Exercises for paretic upper limb after stroke: a combined virtual-reality and telemedicine approach. J Rehabil Med 2009; 41:10161020.

41. Ballester BR, Maier M, María R, et al. Counteracting learned non-use in chronic stroke patients with reinforcementinduced movement therapy. J Neuroeng Rehabil 2016; 13: $1-15$.

42. Shin J-H, Kim M-Y, Lee J-Y, et al. Effects of virtual reality-based rehabilitation on distal upper extremity function and health-related quality of life: A single- blinded, randomized controlled trial. J Neuroeng Rehabil 2016; 13:17.

43. Lee S, Kim Y, Lee B-H. Effect of virtual reality-based bilateral upper extremity training on upper extremity function after stroke: A randomized controlled clinical trial. Occup Ther Int 2016; 23:357-368.
44. Hochstenbach J, Mulder T, Limbeek J Van, et al. Cognitive decline following stroke: A comprehensive study of cognitive decline following stroke. J Clin Exp Neuropsychol 1998; 20:503-517.

45. Holden MK. Virtual environments for motor rehabilitation: Review. Cyberpsychol Behav 2005; 8:187-211.

46. World Health Organization. Towards a Common Language for Functioning, Disability and Health. Geneva. 2002. https:// www.who.int/classifications/icf/icfbeginnersguide.pdf?ua=1 (accessed June 22, 2018).

47. Sullivan KJ, Cen SY. Model of disablement and recovery: Knowledge translation in rehabilitation research and practice. Phys Ther 2011; 91:1892-1904.

48. Subramanian SK, Lourenço CB, Chilingaryan $\mathrm{G}$, et al. Arm motor recovery using a virtual reality intervention in chronic stroke: Randomized control trial. Neurorehabil Neural Repair 2013; 27:13-23.

49. Rand D, Givon N, Weingarden H, ert al. Eliciting upper extremity purposeful movements using video games: A comparison with traditional therapy for stroke rehabilitation. Neurorehabil Neural Repair 2014; 28:733-739.

50. Jo K, Yu J, Jung J. Effects of Virtual Reality-Based Rehabilitation on Upper Extremity Function and Visual Perception in Stroke Patients: a Randomized Control Trial. J Phys Ther Sci. 2012;24:1205-8.

51. Choi Y, Ku J, Lim H, Hyung Kim Y, Paik N. Mobile gamebased virtual reality rehabilitation program for upper limb dysfunction after ischemic stroke. Restor Neurol Neurosci. 2016;34:455-63.

Address correspondence to:

Jose A. Moral-Munoz, PhD

Department of Nursing and Physiotherapy University of Cadiz. Avda. Ana de Viya, 52

Cadiz 11009

Spain

E-mail: joseantonio.moral@uca.es 